\title{
Produção científica da enfermagem brasileira sobre pós-cirúrgico do câncer de próstata
}

\author{
Raquel Juliana de Oliveira Soares, M.Sc.*, Mara Lúcia Amantéa, D.Sc.**, Fátima Cristina Alves Araújo, \\ M.Sc. ${ }^{* * *}$ Priscila de Oliveira Passos****, Amanda de Oliveira Medeiros ${ }^{* * * *}$, Camila Soares Silva ${ }^{* * * *}$
}

\begin{abstract}
*Enfermeira, Docente da Universidade Estácio de Sá/RJ, Pesquisadora do Núcleo de Pesquisa Enfermagem e Saúde do Trabalhador/DESP/EEAN/UFRJ, **Enfermeira, Docente da Universidade Estadual do Rio de Janeiro/UERJ, ***Enfermeira, Docente da Universidade Estácio de SálRJ, ${ }^{* * *}$ Acadêmica de Enfermagem, Universidade Estácio de SálRJ, Campus Norte Shopping Rio de Janeiro/RJ
\end{abstract}

\begin{abstract}
Resumo
Objetivo: Identificar e analisar a produção científica da enfermagem brasileira sobre cuidados de enfermagem ao paciente no pós-cirúrgico de câncer de próstata. Métodos: Revisão de literatura em que foram consultados artigos indexados nas bases de dados Lilacs e BDENF. Resultados: A amostra final desta revisão foi constituída por seis artigos científicos, que de uma forma geral abordaram o cuidado de enfermagem no pós-operatório, enfatizando a assistência de enfermagem para a alta do paciente. A sistematização da assistência de enfermagem constituiu um dos focos de discussão, assim como as orientaçóes dadas aos pacientes sobre autocuidado. Conclusão: Evidenciou-se através desta pesquisa a necessidade dos enfermeiros estarem instrumentalizados para que a assistência ao paciente no pós-cirúrgico do câncer de próstata seja realizada de forma plena, e também a importância de mais estudos acerca da temática visto que somente foram identificados 6 artigos envolvendo a temática, publicados nos últimos dez anos.
\end{abstract}

Palavras-chave: neoplasias da próstata, prostatectomia, cuidados de enfermagem, enfermagem perioperatória.

\section{Abstract \\ Brazilian nursing scientific production on postoperative prostate cancer}

Objective: To identify and analyze the Brazilian nursing scientific production on nursing care after prostate cancer surgery. Methods: This literature review used Lilacs and BDENF data bases to consult indexed articles related to this theme. Results: The final sample of this study was composed of 6 articles related to nursing care after surgery, focusing this care on patient discharge. The systematization of the nursing care constituted one of the discussion topics as well as self-care orientation to patients. Conclusion: This study makes evident that nurses should be well-equipped in order to provide good care to patient after prostate cancer surgery, and also the importance of more studies concerning this theme, due to the fact that we found only six articles published in the last 10 years.

Key-words: prostatic neoplasms, prostatectomy, nursing care, perioperative nursing. 


\section{Resumen}

\section{Producción científica de la enfermería brasileña sobre postoperatorio de cáncer de próstata}

Objetivo: Identificar y analizar la producción científica de la enfermería brasileńa sobre cuidados de enfermería en el postoperatorio de paciente con cáncer de próstata. Métodos: Se trata de una revisión de la literatura que utilizó la base de datos Lilacs e BDENF para consultar artículos sobre el tema. Resultados: La muestra final de esta revisión fue constituida por 06 (seis) artículos científicos que de una forma general abordaron el cuidado de enfermería en el postoperatorio, con énfasis en la atención al paciente para el alta hospitalaria. La sistematización de los cuidados de enfermería constituyó uno de los focos de discusión, así como las orientaciones dadas a los pacientes sobre autocuidado. Conclusión: En este estudio resulta evidente la necesidad de instrumentalizar los enfermeros para que el cuidado al paciente en el posoperatorio de cáncer de próstata sea realizado de forma plena, y también la importancia de más estudios acerca de la temática visto que solamente fueron identificados 06 artículos sobre el tema publicados durante los últimos 10 años.

Palabras-clave: neoplasias de próstata, prostatectomía, cuidados de enfermería, enfermería perioperatoria.

\section{Introdução}

O câncer de próstata é o segundo tipo de neoplasia maligna mais incidente entre os homens brasileiros. No Brasil, a incidência de câncer de próstata é de 51 casos para cada 100.000 homens [1]. Em termos de valores absolutos, o câncer de próstata é o sexto tipo de câncer mais comum no mundo e o mais prevalente entre os homens, representando cerca de $10 \%$ do total de câncer. O número de casos novos diagnosticados de câncer de próstata no mundo é de aproximadamente 543 mil casos por ano, representando $15,3 \%$ de todos os casos incidentes de câncer em países desenvolvidos e 4,3\% dos casos em países em desenvolvimento [1].

Neste contexto, após o diagnóstico do câncer, as opçóes de tratamento serão baseadas no estágio da doença, idade, e sintomas do paciente, cuja probabilidade de cura é alta desde que o diagnóstico seja realizado em um estágio inicial da doença [2]. Em estágios mais avançados, as opções de tratamento se voltam para os procedimentos cirúrgicos.

Para assistir o paciente com câncer de próstata, os enfermeiros seguem protocolos institucionais, sistematizando assim o seu cuidado, porém estes profissionais devem compreender que realizar procedimentos no paciente é diferente de realizar procedimentos para e com o paciente, pois os pacientes submetidos a uma cirurgia geniturinária podem desenvolver várias reaçóes, como sentimento de medo, raiva, desamparo, tristeza, vergonha, entre outros [3]. Neste sentido, a falta da interação enfermeiro-paciente pode resultar em danos ao paciente, além de contribuir para o desprestígio da profissão de enfermagem [4].
Os enfermeiros desempenham um importante papel no período pós-cirúrgico, não só com relação aos procedimentos técnicos, mas também no que tange as orientaçóes ao paciente e a família, principalmente no preparo destes pacientes para a alta, uma vez que frequentemente, estes deixam o hospital com dúvidas e expectativas, sobretudo com relação ao funcionamento do aparelho urinário e reprodutor [5].

Além de a complexidade que envolve o conhecimento das necessidades específicas que podem apresentar os homens submetidos à prostatectomia e a importância das açóes de cuidado de enfermagem em seu preparo para o pós-cirúrgico e a alta hospitalar [5], a insuficiência de publicaçóes sobre a temática justifica a realização deste estudo.

Diante da importância de instrumentalizar os enfermeiros para uma assistência sistematizada e humanizada, o presente estudo objetivou identificar a produção científica da enfermagem brasileira sobre os cuidados dos enfermeiros ao paciente no pós-cirúrgico de câncer de próstata.

\section{Métodos}

Para a elaboração da presente revisão as seguintes etapas foram percorridas: identificação do tema e formulação da questão norteadora; determinação dos critérios de inclusão e exclusão de artigos (seleção da amostra); definiçáo das informaçóes a serem extraídas dos artigos; avaliaçáo dos resultados incluídos na revisão; interpretação dos resultados.

Esta revisão bibliográfica teve como tema a assistência dos enfermeiros no pós-cirúrgico do câncer de próstata, sendo apoiada na seguinte 
questáo norteadora: quais os cuidados dos enfermeiros direcionados aos pacientes no pós-cirúrgico do câncer de próstata são apontados na literatura brasileira? Os critérios de inclusão definidos para a presente revisão foram: artigos publicados na íntegra em português, no período compreendido entre os anos 2000 a 2010, sendo pelo menos um dos autores enfermeiro. Sobre os critérios de exclusão foram definidos: artigos que não apresentavam o profissional enfermeiro como autor, artigos que apresentavam somente a publicação do resumo. Estes critérios foram traçados pautados nos objetivos do estudo, sendo importantes para os resultados do estudo. Para a coleta de dados, foi elaborado um instrumento que contemplou os seguintes itens: identificação do artigo; autores; objetivos do estudo; metodologia; principais achados; considerações finais; periódico e o ano da publicação. O levantamento dos dados se deu no período de agosto a outubro do ano 2010.

Para a seleção dos artigos foi utilizada a Biblioteca Virtual em Saúde (BVS). Os descritores utilizados na busca dos dados foram neoplasias da próstata, prostatectomia, enfermagem perioperatória e enfermagem em pós-anestésico. A referida busca foi realizada pelo acesso on-line, utilizando os critérios de inclusão, sendo a amostra final da pesquisa constituída 6 artigos. Dos 6 artigos, 5 foram encontrados na base de dados Lilacs (Literatura Latino-Americana e do Caribe em Ciências da Saúde) e 1 foi encontrado na base BDENF (Base de dados de enfermagem).

A apresentação e discussão dos resultados foram feitos de forma descritiva, possibilitando a avaliação da aplicabilidade da revisão elaborada, de forma a atingir o objetivo desse método, ou seja, impactar positivamente na qualidade da prática de enfermagem, fornecendo subsídios ao enfermeiro na sua tomada de decisão.

\section{Resultados}

A amostra final desta revisão foi constituída por 6 artigos científicos, selecionados pelos critérios de inclusão previamente estabelecidos. A apresentação dos resultados está dividida em dois segmentos: o primeiro com uma tabela contemplando as informaçôes: autor, ano da publicação, periódico publicado e a metodologia abordada nos estudos, e o segundo com a síntese dos artigos pesquisados, com o título, o objetivo e as principais consideraçóes dos estudos.

Tabela I - Distribuição dos artigos segundo autor, ano de publicação, local de publicação e metodologia abordada nos estudos.

\begin{tabular}{|c|c|c|c|}
\hline Autor & Ano & Local de Publicação & Metodologia \\
\hline Santana MS et al. [6] & 2005 & Revista Nursing - São Paulo/SP & Não especifica no resumo \\
\hline Napoleão AA et al. [7] & 2009 & $\begin{array}{l}\text { Revista Eletrônica de Enfermagem - } \\
\text { Goiânia/GO }\end{array}$ & Pesquisa qualitativa, descritiva \\
\hline Vianna MC [8] & 2009 & $\begin{array}{l}\text { Revista Ciência, Cuidado e Saúde (on } \\
\text { line) - Maringá/PR }\end{array}$ & Artigo de Reflexão \\
\hline Napoleão M et al. [8] & 2010 & $\begin{array}{l}\text { Revista Acta Paulista de Enfermagem - } \\
\text { São Paulo/SP }\end{array}$ & Revisão Integrativa da literatura \\
\hline Stumm EMF et al. [9] & 2010 & $\begin{array}{l}\text { Revista Eletrônica Textos e Contextos - } \\
\text { Porto Alegre/RS }\end{array}$ & Pesquisa qualitativa, descritiva \\
\hline Napoleão AA et al. [10] & 2010 & $\begin{array}{l}\text { Revista de Enfermagem UFPE (on line) } \\
\text { - Recife/PE }\end{array}$ & Revisão Bibliográfica \\
\hline
\end{tabular}

Verificou-se que a maior parte dos artigos foi publicada recentemente (2009 e 2010) demonstrando mesmo que ainda incipiente a maior preocupação dos profissionais com a temática nos últimos anos. A abordagem qualitativa predominou nos estudos analisados. Merece destaque o fato de um dos resumos não explicitar qual o enfoque metodológico utilizado. Com relação ao local de publicação, dos 6 artigos encontrados, 5 foram publicados em Re- vistas Gerais de Enfermagem e 1 foi publicado em Revista do Serviço Social. Náo foram encontrados no período estudado mais de um artigo publicado nas referidas revistas, com a temática investigada.

$\mathrm{Na}$ síntese dos artigos estudados apresentamos: título, objetivo(s) e as principais consideraçôes.

1) Santana MS, Garcia TR [6]. Diagnóstico de enfermagem em pacientes submetidos à prostatectomia. Objetivo: Estabelecer um perfil diag- 
nóstico de enfermagem de pacientes submetidos à prostatectomia, utilizando-se a taxonomia II da NANDA International. Principais consideraçóes: os dados coletados permitiram mais de 90 afirmativas diagnósticas por momento de observação, com uma média de três diagnósticos de enfermagem por paciente, demonstrando sua importância no processo assistencial de enfermagem direcionado a essa clientela.

2) Napoleão AA et al. [7]. Diagnósticos de enfermagem para o planejamento de altas de homens prostatectomizados: um estudo preliminar. $\mathrm{Ob}$ jetivo: identificar diagnósticos de enfermagem em pacientes prostatectomizados com vistas a oferecer subsídios para a elaboração de planos de cuidados para a alta hospitalar. Principais consideraçôes: Os diagnósticos identificados em todos os pacientes foram conhecimento deficiente (acerca dos cuidados pós-operatórios e com sonda vesical de demora) risco de volume de líquidos deficiente, risco de lesáo, risco de infecção, integridade tissular prejudicada. Menos frequentemente foram identificados: ansiedade, risco de baixa autoestima situacional, disposição para bem-estar espiritual aumentado, mobilidade física prejudicada, recuperaçáo cirúrgica retarda$\mathrm{da}$, risco de sentimento de impotência e risco de integridade da pele prejudicada. Os diagnósticos elaborados possibilitaram um detalhamento das condiçóes dos sujeitos, favoreceram a utilização de uma abordagem individualizada, a identificação de importantes necessidades para a alta e puderam oferecer um adequado embasamento para a elaboraçáo de planos de cuidados.

3) Vianna MC, Napoleão AA [8]. Reflexóes sobre cuidados de enfermagem para alta de paciente prostatectomizados. Objetivo: apresentar uma reflexão sobre a importância da implementação de cuidados específicos de enfermagem no preparo de pacientes prostatectomizados para a alta. Principais consideraçôes: Destaca-se a importância de assegurar uma atuação efetiva da enfermagem no preparo dos pacientes para o autocuidado após a alta, o que pode contribuir para a redução de sua ansiedade, maior segurança no autocuidado e sua melhor recuperação.

4) Napoleão AA, Mata LRF [5]. Intervenções de Enfermagem para alta de paciente prostatectomizado: revisão integrativa. Objetivo: identificar o conhecimento que se tem produzido sobre intervençóes de enfermagem, na literatura cien- tífica da enfermagem, com vistas ao preparo do paciente prostatectomizado para alta hospitalar. Principais consideraçóes: Destaca-se a importância da realização de estudos experimentais e quase experimentais sobre a eficácia da informação para o autocuidado aos pacientes e suas famílias, melhores cuidados de enfermagem na incontinência urinária e disfunção erétil e diagnósticos de enfermagem específicos para orientar planos de cuidados de enfermagem a esses pacientes.

5) Stumm EMF et al. [9]. Vivências de idosos submetidos à prostatectomia por câncer: subsídios para o cuidado de enfermagem. Objetivo: apreender vivências de idosos submetidos à cirurgia de prostatectomia por câncer. Principais consideraçóes: Ressalta-se o papel que a equipe de enfermagem desempenha no cuidado ao idoso oncológico, principalmente diante da necessidade que muitos têm em dialogar e esclarecer dúvidas referentes à doença. Nesse sentido, destaca-se a importância do enfermeiro possuir conhecimentos técnicos e científicos, habilidade para transmitir esses conhecimentos, aliada à sensibilidade para saber ouvir e interpretar o que o idoso quer dizer e, a partir daí, proporcionar apoio emocional tanto a ele quanto aos familiares, no enfrentamento de todo o processo da doença.

6) Napoleão AA et al. [10]. Aplicabilidade da Classificação das Intervençóes de Enfermagem (NIC) no preparo para a alta de pacientes prostatectomizados. Objetivo: identificar a aplicabilidade da NIC na elaboraçáo de planos de cuidados para alta de pacientes prostatectomizados. Principais consideraçóes: a NIC pode ser aplicável na elaboraçáo de planos de cuidado para alta desses pacientes, no entanto, não apresenta intervençóes específicas a esta situação. Uma vez que apresenta intervenções e atividades aplicáveis ou não, faz-se necessário conhecer sua estrutura taxonômica e, obrigatoriamente, as necessidades específicas dos pacientes para que sejam feitas escolhas adequadas ao plano de cuidados, o que é positivo na medida em que favorece a sistematização da assistência com uso de uma linguagem padronizada, além de uma abordagem individualizada e que considera necessidades específicas dos pacientes.

\section{iscussão}

Evidenciou-se que os 6 estudos abordaram a preocupação dos autores com relaçáo aos cuidados de 
enfermagem para a alta de pacientes prostatectomizados. A seguir, são apresentados os temas que emergiram dos artigos examinados e respectiva análise.

\section{Diagnóstico de Enfermagem para a elaboração do plano de cuidado}

Dos estudos pesquisados, 2 enfatizaram a importância do diagnóstico de enfermagem para a elaboraçáo do plano de cuidado para o paciente no pós-cirúrgico de prostatectomia. Os estudos mostram como é importante o enfermeiro analisar e realizar o exame físico para assim conhecer o paciente e entender sua patologia conseguindo, dessa forma, traçar um plano assistencial que minimize efeitos maléficos que possam prejudicar o bem estar físico, psíquico e social. $\mathrm{O}$ processo de enfermagem tem representado o principal instrumento metodológico para o desempenho sistemático da prática profissional. Por diagnóstico de enfermagem, entende-se uma etapa desse processo, responsável por fornecer meios para propor intervenções de responsabilidade exclusiva do enfermeiro quanto aos problemas de saúde detectados. Além de ser instrumento de trabalho desses profissionais, proporciona o uso de linguagem própria e facilita a comunicação com os pacientes [11].

\section{Autocuidado do paciente no pós-cirúrgico de prostatectomia}

O autocuidado é um comportamento que implica no papel ativo do cliente em prática de atividades que o indivíduo desempenha em seu próprio benefício, a fim de manter a vida, a saúde e o bem estar [12]. Desta forma, dois dos estudos pesquisados evidenciaram a importância do autocuidado, principalmente após a alta da cirurgia de prostatectomia, em que a enfermeira tem um papel fundamental, no que tange as orientaçóes do paciente e sua família. A enfermagem atua de forma direta no que diz respeito às informações necessárias para que o paciente possa ter autonomia e confiança no seu próprio cuidado; atuando desta forma será alcançado êxito e diminuirá medos e ansiedades possíveis causadas pela doença. Neste sentido, a enfermeira assume o compromisso de compartilhar conhecimentos, dividindo o espaço com aquele que é o sujeito e não mais o objeto das ações do cuidado, visando assim uma melhor qualidade de vida deste paciente.

\section{Sistematizaçáo da Assistência de Enfermagem}

A Sistematização da Assistência de Enfermagem (SAE) é o modelo metodológico ideal para o enfermeiro aplicar seus conhecimentos técnico-científicos na prática assistencial, favorecendo o cuidado e a organização das condiçóes necessárias para que ele seja realizado [13]. Quanto maior o número de necessidades afetadas do cliente, maior é a necessidade de se planejar a assistência, uma vez que a sistematizaçáo das açôes visa à organização, à eficiência e à validade da assistência prestada. Desta forma, destaca-se que um dos estudos pesquisado relatou a Sistematização da Assistência de Enfermagem (SAE), traçando as principais etapas que o enfermeiro deve seguir para que assim consiga realizar um cuidado padronizado e eficaz relacionado ao câncer de próstata, garantindo um bom prognóstico para o paciente prostatectomizado. Para o paciente no pós-cirúrgico do câncer de próstata, dada a sua situaçáo, a assistência de enfermagem sistematizada é ainda mais necessária, pois facilitará o domínio apurado da técnica, conciliando-o com um cuidado humanizado e holístico.

\section{Conhecimento dos enfermeiros acerca do câncer de próstata e suas complicaçóes}

Em um dos estudos foi abordada a importância do enfermeiro no domínio da patologia e suas complicaçóes para o auxílio e esclarecimento aos idosos. Esse profissional deve estar o mais próximo ao paciente para conseguir laços de confiança e respeito, já que está doença é algo extremamente íntimo, uma vez que mexe com o lado sexual masculino, o que para o homem é algo delicado, e sendo em pessoas idosas em alguns casos é mais constrangedor. Destaca-se ainda a importância do conhecimento científico e a formação específica do enfermeiro que atua na área da oncologia, tendo desta forma melhores condiçóes de traçar planos de cuidados específicos a estes pacientes.

Os enfermeiros que cuidam de pacientes oncológicos trabalham em um ambiente de grande exigência, seja pela alta complexidade do paciente, seja pelas manifestaçóes de cada indivíduo, e isto faz com que os enfermeiros estejam em constante atualizaçáo de conhecimentos. Cabe a esses profissionais a sensibilidade de perceber, de captar no paciente o que realmente ele compreendeu das orientaçóes que lhe foram transmitidas [9] e se há dúvidas que possam ser sanadas antes da alta hospitalar. 


\section{Conclusão}

A partir deste estudo identificamos a escassez de publicaçóes envolvendo cuidados de enfermagem no pós-cirúrgico de câncer da próstata, visto que somente foram identificados 6 artigos envolvendo a temática, publicados nos últimos 10 anos. Tais artigos identificados abordavam o assunto sob diferentes ângulos passando pelas vivências de idosos submetidos à cirurgia de prostatectomia; diagnóstico de enfermagem voltados a elaboração de plano de cuidados pós-alta; conhecimentos produzidos sobre intervenções de enfermagem com vista ao preparatório do paciente prostatectomizado para a alta, além de reflexão sobre cuidados específicos referente a esta temática.

A partir dos artigos estudados ressalta-se o papel da equipe de enfermagem diante da necessidade de diálogo e esclarecimento de dúvidas não só do paciente, mas também de sua família. Destaca-se a importância dos conhecimentos técnicos e científicos aliados à habilidade para transmiti-los, além de sensibilidade para saber ouvir e interpretar as demandas da clientela. Reforça-se a importância da realização de estudos sobre a eficácia de informaçôes para o autocuidado, incontinência urinária e disfunção erétil; além de estudos que envolvam diagnósticos de enfermagem específicos para a temática pós-cirúrgico de prostatectomia.

\section{Referências}

1. Brasil. Ministério da Saúde. Instituto Nacional do Câncer. Estimativa 2010: incidência de câncer no Brasil / Instituto Nacional de Câncer. Rio de Janeiro: INCA; 2009.

2. Smeltzer CS, Bare GB. Tratado de Enfermagem Médico Cirúrgico. 10a ed. Rio de Janeiro: Guanabara Koogan; 2005.

3. Nagle GM. Cirurgia geniturinária. In: Meeker $\mathrm{MH}$, Rothrock JC. Alexander. Cuidados de enfermagem ao paciente cirúrgico. 10a ed. Rio de Janeiro: Guanabara Koogan; 1997.

4. Waldow VR. Cuidado humano: o resgate necessário. 3aed. Porto Alegre: Sagra; 2001.

5. Mata LRF, Napoleão AA. Intervençóes de enfermagem para alta de paciente prostatectomizado: revisão integrativa. Acta Paul Enferm 2010;23(4):574-9.

6. Santana MS, Garcia TR. Diagnósticos de enfermagem em pacientes submetidos a prostatectomia. Nurs 2005;90(8):528-33.

7. Napoleão AA, Caldato VG, Petrilli Filho JF. Diagnósticos de enfermagem para o planejamento da alta de homens prostatectomizados: um estudo preliminar. Rev Eletrônica Enferm 2009;11(2):286-94.

8. Vianna MC, Napoleão AA. Reflexóes sobre cuidados de enfermagem para a alta de pacientes prostatectomizados. Ciênc Cuid Saúde 2009;8(2):269-73.

9. Stumm EMF, Scherer JA, Kirchner RM, Berlezi EM, Bento LB. Vivências de idosos submetidos à prostatectomia por câncer: subsídios para o cuidado de enfermagem. Texto \& Contexto Enferm 2010;9:89-102.

10. Napoleão AA, Mata LRF, Vianna MC, Rodrigues RL. Aplicabilidade da Classificação das Intervençóes de Enfermagem (NIC) no preparo para alta de pacientes prostatectomizados. Rev Enferm UFPE 2010;4(1):316-23.

11. Costa AGS, Oliveira ARS, Alves FEC, Chaves DBR, Moreira RP, Araújo TL. Diagnóstico de enfermagem: mobilidade física prejudicada em pacientes acometidos por acidente vascular encefálico. Rev Esc Enferm USP 2010;44(3): 753-8.

12. Dupas G, Pinto IC, Mendes MD, Benedini Z. Reflexão e síntese acerca do modelo auto cuidado de Orem. Acta Paul Enf 1997;7(1):19-26.

13. Bittar DB, Pereira LV, Lemos RCA. Sistematização da assistência de enfermagem ao paciente crítico: proposta de instrumento de coleta de dados. Texto Contexto Enferm 2006;15(4):617-28. 